\title{
Erratum to: Causes and Treatment of Recurrent Hyperparathyroidism After Subtotal Parathyroidectomy in the Presence of Multiple Endocrine Neoplasia 1
}

Maria D. Balsalobre Salmeron • Jose Manuel Rodriguez Gonzalez • Joan Sancho Insenser - Albert Goday - Nuria Maria Torregrosa Perez • Antonio Rios Zambudio • Pascual Parrilla Paricio - Antonio Sitges Serra

Published online: 18 May 2010

(C) Société Internationale de Chirurgie 2010

Erratum to: World J Surg (2010) 34(6):1325-1331

DOI 10.1007/s00268-010-0605-2

Author Joan Sancho Insenser's family name appears incorrectly in the original article.

The online version of the original article can be found under doi:10.1007/s00268-010-0605-2.

M. D. Balsalobre Salmeron · J. M. Rodriguez Gonzalez ( $₫)$.

N. M. Torregrosa Perez · A. Rios Zambudio · P. Parrilla Paricio

Unit Endocrine Surgery, Hospital Universitario Virgen de la

Arrixaca, Murcia, Spain

e-mail: jmrodri@um.es

J. Sancho Insenser - A. Goday - A. Sitges Serra

Unit Endocrine Surgery, Hospital Universitario del Mar,

Barcelona, Spain 